Supporting Information

\title{
Structure-Based Relative Energy Prediction Model: A \\ Case Study of Pd(II) Catalyzed Ethylene Polymerization and the Electronic Effect of Ancillary Ligands
}

\author{
Han Lua , Xiaohui Kang ${ }^{\mathrm{c}}$, Yi Luo $\mathrm{ab}^{*}$ \\ a State Key Laboratory of Fine Chemicals, School of Chemical Engineering, Dalian University of Technology, \\ Dalian, 116024, China; \\ ${ }^{b}$ PetroChina Petrochemical Research Institute, Beijing 102206, China. \\ ' College of Pharmacy, Dalian Medical University, Dalian, 116044, China; \\ *E-mail: luoyi@dlut.edu.cn
}

Part: Page:

$\begin{array}{ll}\text { 1. Data set } & \text { S2 }\end{array}$

$\begin{array}{ll}\text { 2. Model formulation and validation } & \text { S4 }\end{array}$

3. Testing of the unknow ligands $\quad$ S6

4. Models constructed by the geometrical parameters of agostic species S7

5. Modeling of the energy profile with propyl chain $\quad$ S9

$\begin{array}{ll}\text { 6. Electronic effect of ancillary ligands } & \text { S10 }\end{array}$

$\begin{array}{ll}\text { 7. Coefficient analysis of the models } & \text { S12 }\end{array}$

8. Discussion of the symmetric of the ancillary ligands $\quad$ S15

9. Discussion of the ligand backbone $\quad$ S16

$\begin{array}{ll}\text { Reference } & \text { S17 }\end{array}$ 


\section{Data set}

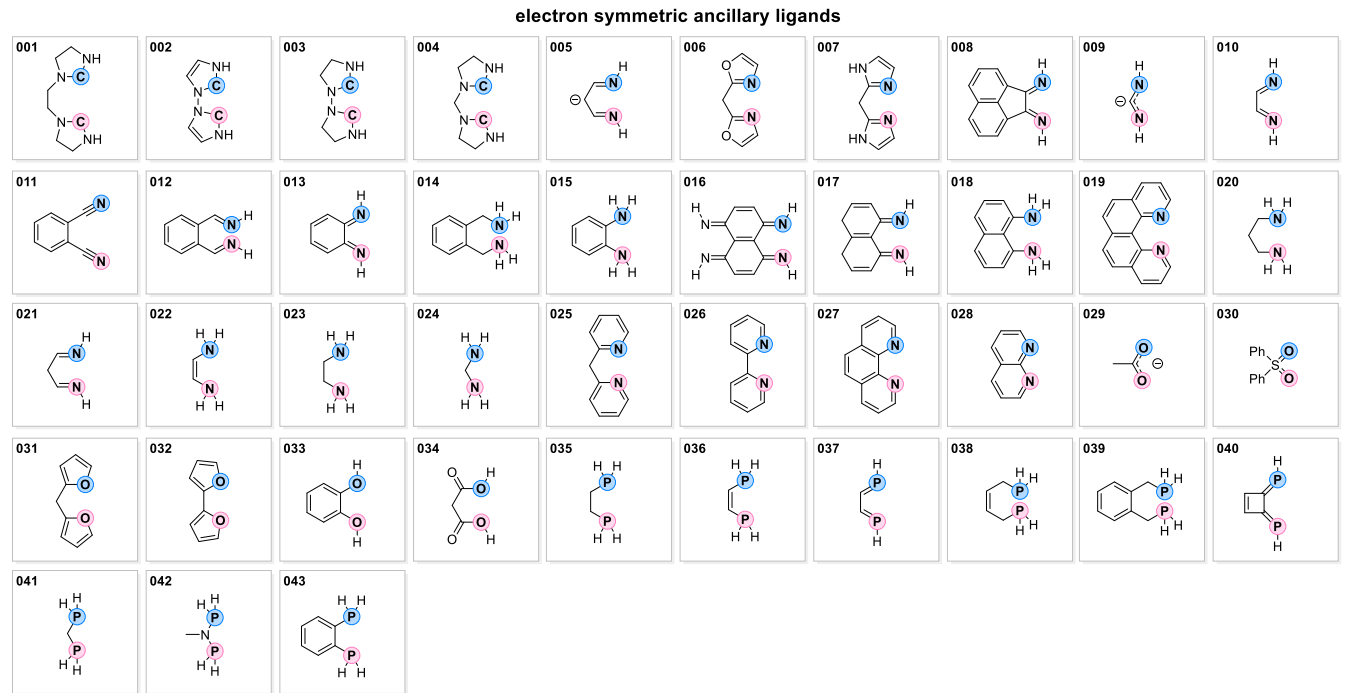

(1)

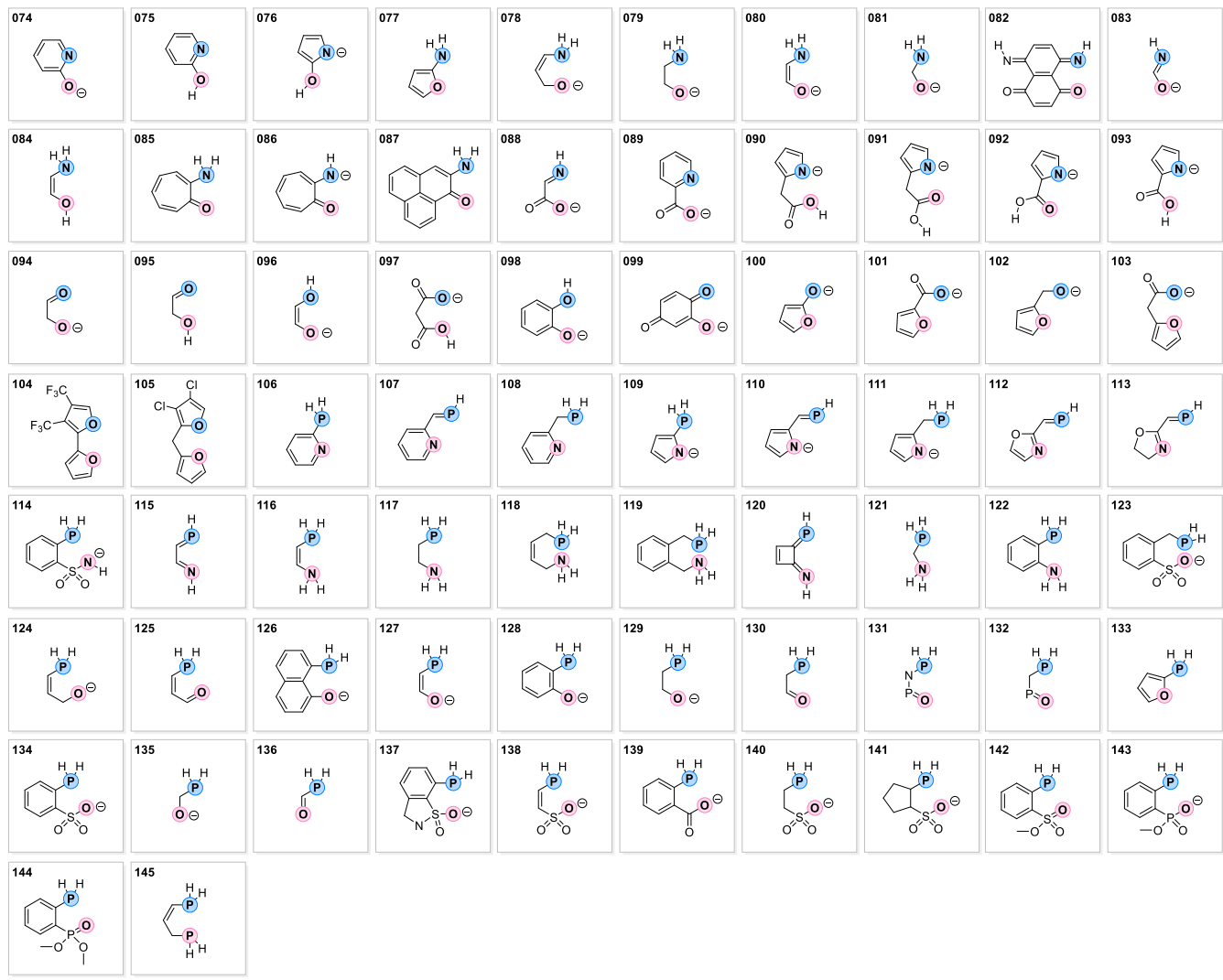

Figure S1. The $145(43+102)$ auxiliary ligands in training set that contain P, N, O and carbene coordinating atoms. 

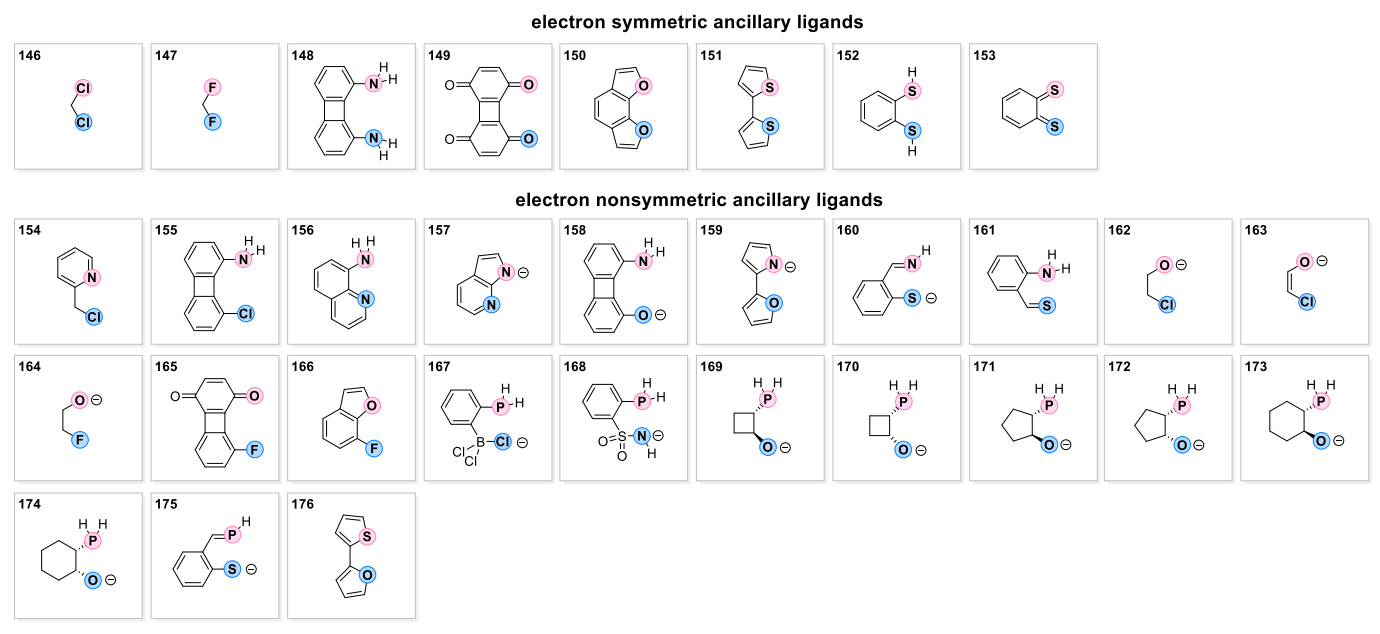

Figure S2. The $31(8+23)$ auxiliary ligands in testing set that contain S, F, Cl coordinating atoms. 


\section{Model formulation and validation}

Table S1. The six linear models based on the structure and energy of initial species with methyl group

\begin{tabular}{|c|c|}
\hline Model & Equation \\
\hline 1 & $\mathbf{y}_{1}=21.5+1.78 \mathbf{x}_{4}-67.2 \mathbf{x}_{5}+125 \mathbf{x}_{6}-1.30 \mathbf{x}_{8}$ \\
\hline 2 & $\mathbf{y}_{2}=-0.4-90.1 \mathbf{x}_{2}+1.53 \mathbf{x}_{4}-65.4 \mathbf{x}_{5}+137 \mathbf{x}_{6}-0.909 \mathbf{x}_{8}$ \\
\hline 3 & $\mathbf{y}_{3}=5.6-39.6 \mathbf{x}_{1}+61.1 \mathbf{x}_{5}+0.548 \mathbf{x}_{8}$ \\
\hline 4 & $\mathbf{y}_{4}=-34.8-26.9 \mathbf{x}_{1}-251 \mathbf{x}_{3}+23.5 \mathbf{x}_{6}-310 \mathbf{x}_{7}-0.900 \mathbf{x}_{8}$ \\
\hline 5 & $\mathbf{y}_{5}=0.0-112 \mathbf{x}_{2}+2.34 \mathbf{x}_{4}+112 \mathbf{x}_{6}-2.34 \mathbf{x}_{8}$ \\
\hline 6 & $\mathbf{y}_{6}=0.0-66.2 \mathbf{x}_{1}+117 \mathbf{x}_{2}+66.2 \mathbf{x}_{5}-117 \mathbf{x}_{6}$ \\
\hline
\end{tabular}
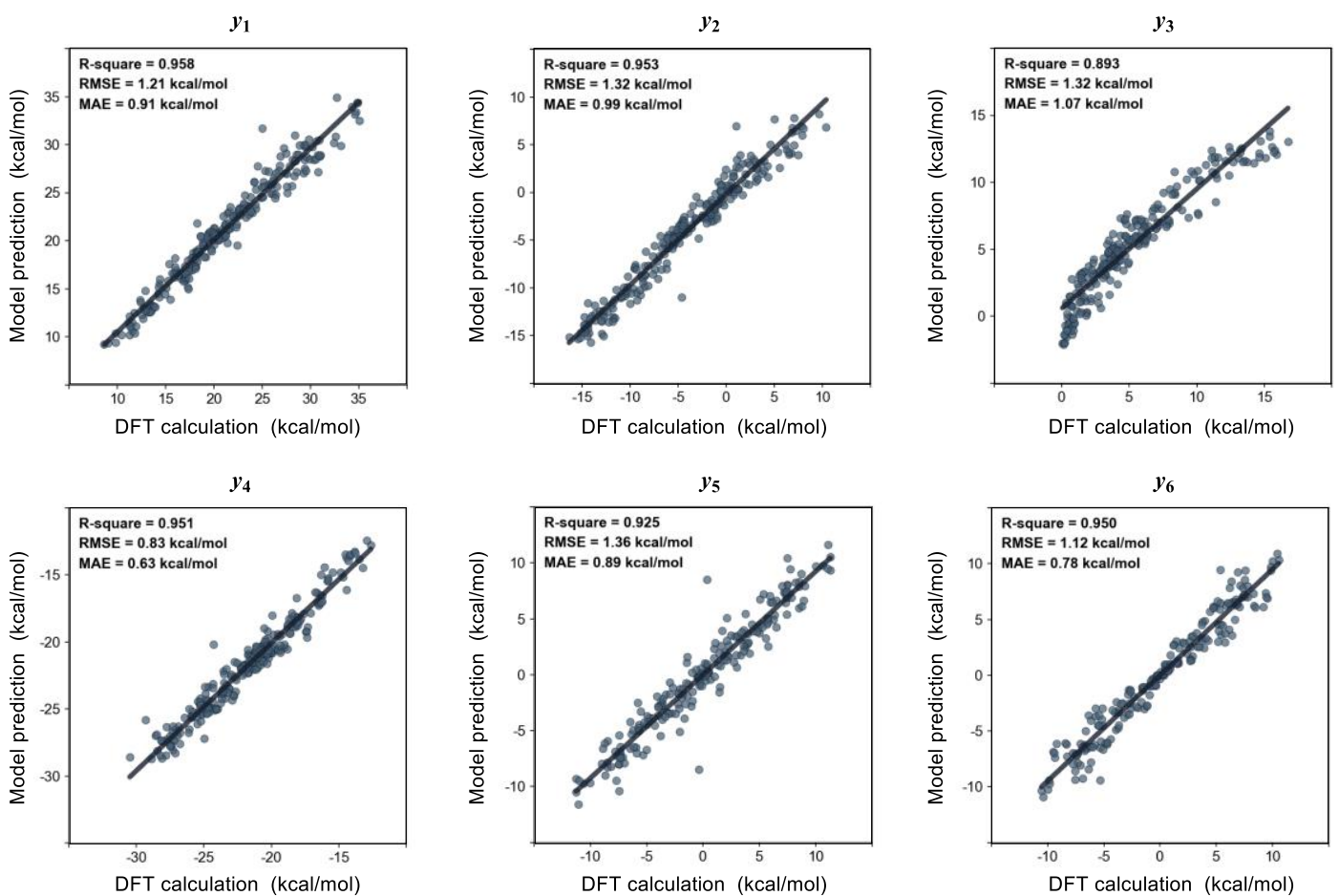

Figure S3. A comparison of the DFT calculated energy and model predicted energy value of 6 models shown in Table S1. 
$y_{1}$

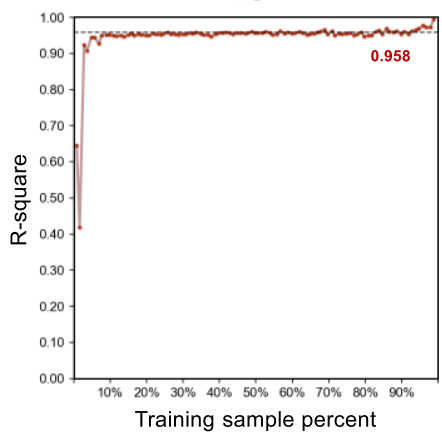

$y_{4}$

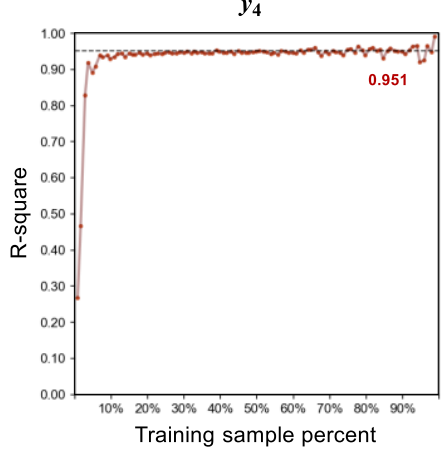

$y_{2}$

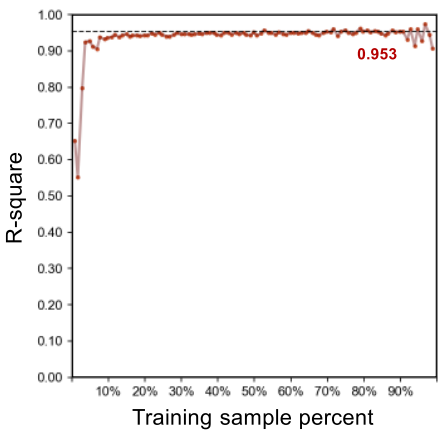

$y_{5}$

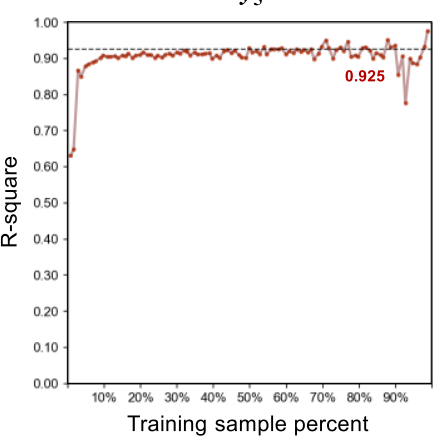

$y_{3}$

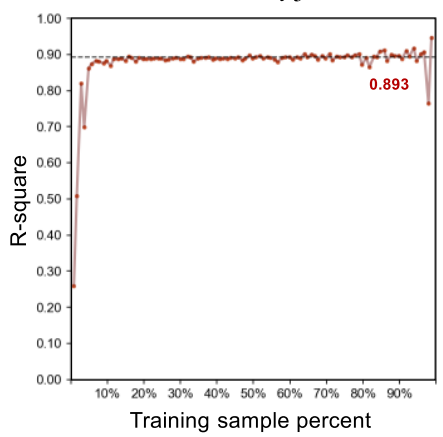

$y_{6}$

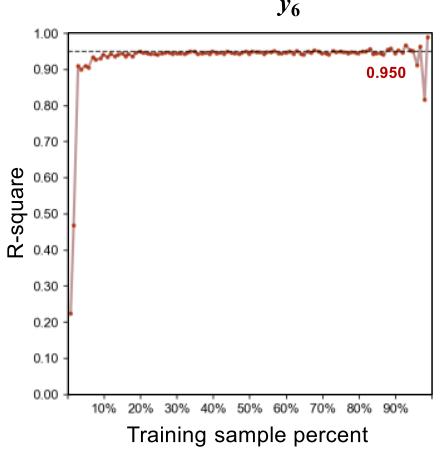

Figure S4. Internal cross-validation results of the 6 models.
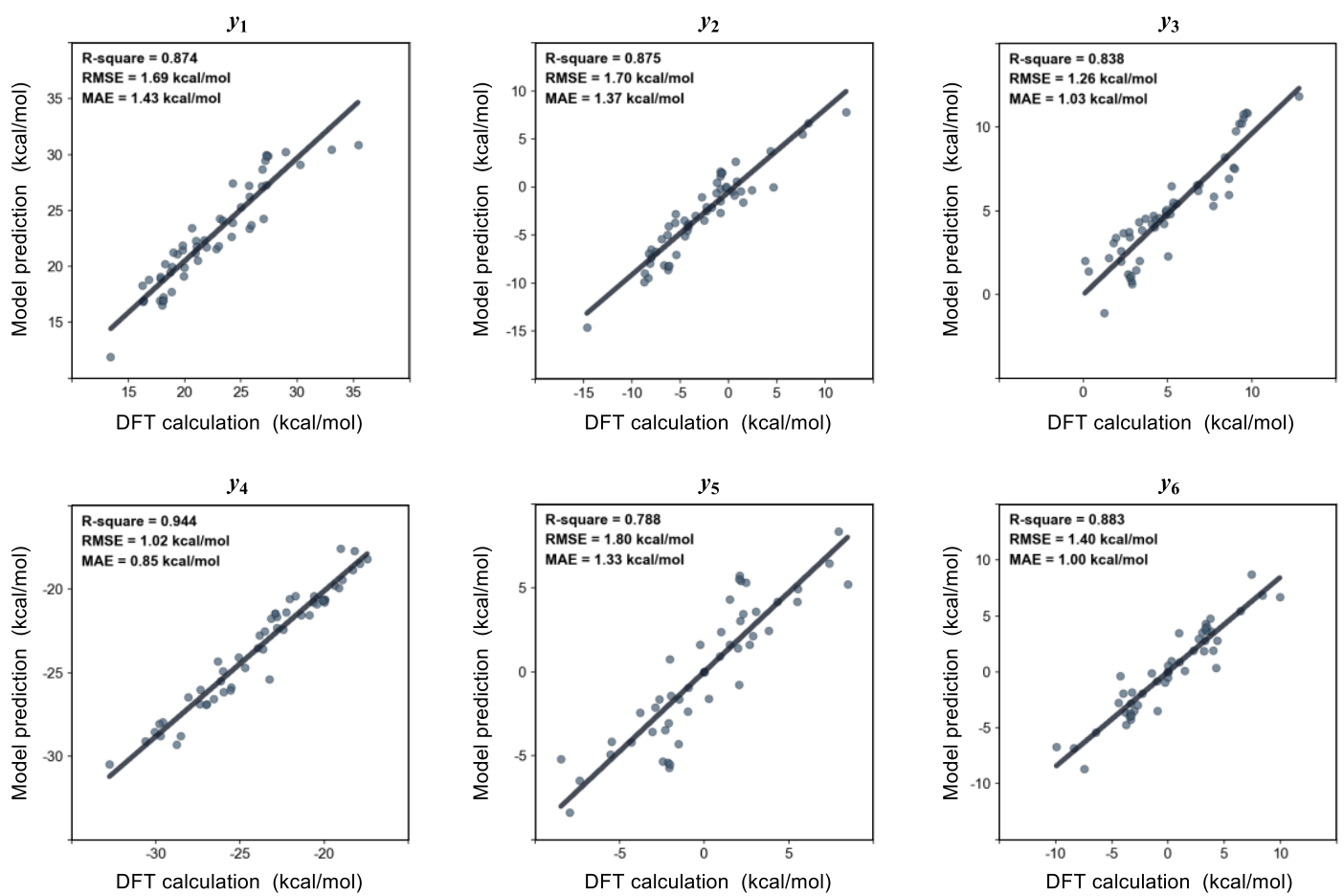

Figure S5. External validation of the 6 models by using 54 testing samples. 


\section{Testing of the unknow ligands}

Table S2. Model performance on two catalysts with ancillary ligands containing hemilabile side arm.

\begin{tabular}{|c|c|c|c|c|c|c|c|c|}
\hline sample & level & $\begin{array}{c}\mathrm{y} 1 \\
\mathrm{kcal} / \mathrm{mol}\end{array}$ & $\begin{array}{c}\mathrm{y} 2 \\
\mathrm{kcal} / \mathrm{mol}\end{array}$ & $\begin{array}{c}\mathrm{y3} \\
\mathrm{kcal} / \mathrm{mol}\end{array}$ & $\begin{array}{c}\mathrm{y} 4 \\
\mathrm{kcal} / \mathrm{mol}\end{array}$ & $\begin{array}{c}\text { y5 } \\
\mathrm{kcal} / \mathrm{mol}\end{array}$ & $\begin{array}{c}\text { y6 } \\
\mathrm{kcal} / \mathrm{mol}\end{array}$ & $\begin{array}{c}\text { RMSE } \\
\mathrm{kcal} / \mathrm{mol}\end{array}$ \\
\hline & calculation & 20.3 & -4.0 & 3.3 & -21.2 & 0.6 & -0.8 & \multirow{4}{*}{0.5} \\
\hline & prediction & 21.4 & -3.5 & 3.8 & -20.8 & 0.9 & -0.7 & \\
\hline \multirow{2}{*}{ (cis) } & calculation & 18.7 & -5.4 & 4.4 & -20.5 & -0.6 & 0.8 & \\
\hline & prediction & 19.8 & -5.1 & 4.6 & -20.9 & -0.9 & 0.7 & \\
\hline $\mathrm{N}(\mathrm{Et})_{2}$ & calculation & 20.7 & -3.5 & 3.3 & -21.4 & 0.8 & -1.0 & \multirow{4}{*}{0.6} \\
\hline & prediction & 21.7 & -3.2 & 3.9 & -20.8 & 1.2 & -0.6 & \\
\hline \multirow{2}{*}{$=\quad$ (cis) } & calculation & 18.8 & -5.3 & 4.4 & -20.5 & -0.8 & 1.0 & \\
\hline & prediction & 19.8 & -5.1 & 4.5 & -21.1 & -1.2 & 0.6 & \\
\hline
\end{tabular}

Table S3. Model performance on two catalysts with monodentate ancillary ligands.

\begin{tabular}{|c|c|c|c|c|c|c|c|c|}
\hline sample & level & $\begin{array}{c}\mathrm{yl} \\
\mathrm{kcal} / \mathrm{mol}\end{array}$ & $\begin{array}{c}\mathrm{y} 2 \\
\mathrm{kcal} / \mathrm{mol}\end{array}$ & $\begin{array}{c}\mathrm{y} 3 \\
\mathrm{kcal} / \mathrm{mol}\end{array}$ & $\begin{array}{c}\mathrm{y} 4 \\
\mathrm{kcal} / \mathrm{mol}\end{array}$ & $\begin{array}{c}\mathrm{y5} \\
\mathrm{kcal} / \mathrm{mol}\end{array}$ & $\begin{array}{c}\text { y6 } \\
\mathrm{kcal} / \mathrm{mol}\end{array}$ & $\begin{array}{c}\text { RMSE } \\
\mathrm{kcal} / \mathrm{mol}\end{array}$ \\
\hline \multirow{2}{*}{$\begin{array}{c}(\mathrm{Ph})_{3} \mathrm{P} \\
\\
\ominus \mathrm{Cl}\end{array}$} & calculation & 24.5 & -3.4 & 2.6 & -20.0 & 2.2 & -7.0 & \multirow{4}{*}{1.6} \\
\hline & prediction & 26.0 & -3.0 & -1.2 & -19.3 & 0.8 & -6.7 & \\
\hline \multirow{2}{*}{$\begin{array}{cc}\ominus \mathrm{Cl} & \\
(\mathrm{Ph})_{3} \mathrm{P} & \text { (cis) }\end{array}$} & calculation & 17.1 & -12.5 & 13.0 & -15.1 & -2.2 & 7.0 & \\
\hline & prediction & 17.0 & -10.5 & 10.8 & -15.6 & -0.8 & 6.7 & \\
\hline$v^{-}$ & calculation & 31.0 & 2.8 & 1.3 & -19.4 & 8.4 & -7.7 & \multirow{4}{*}{2.0} \\
\hline$\Theta \mathrm{Cl}$ & prediction & 29.6 & 1.5 & -1.1 & -20.5 & 4.5 & -8.2 & \\
\hline \multirow{2}{*}{$\begin{array}{l}\Theta \mathrm{Cl} \\
\widehat{N}_{-} \cdot \text { (cis }\end{array}$} & calculation & 15.0 & -13.3 & 12.3 & -19.8 & -8.4 & 7.7 & \\
\hline & prediction & 15.8 & -11.2 & 11.8 & -17.8 & -4.5 & 8.2 & \\
\hline
\end{tabular}


4. Models constructed by the geometrical parameters of agostic species

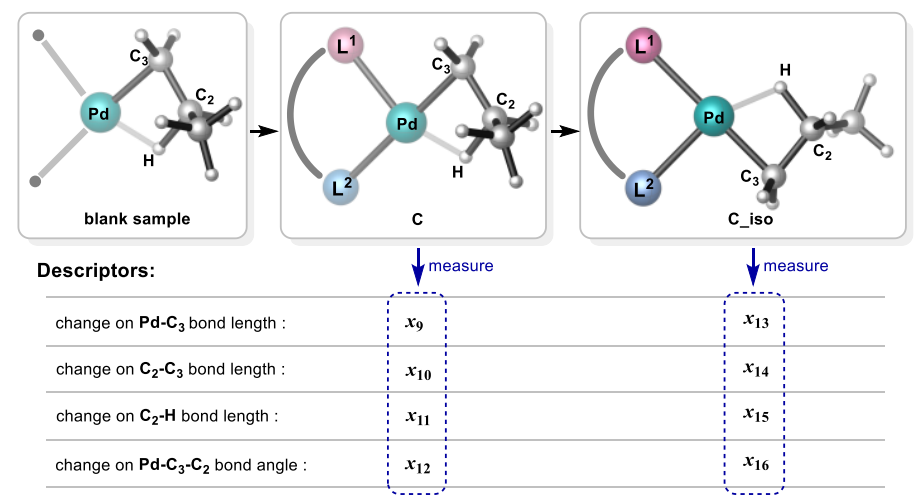

Figure S6. Geometrical parameter changes as the descriptors of the involved agostic species relative to corresponding blank sample.

Table S4. The six linear models based on the structure of the agostic species.

\begin{tabular}{|c|c|}
\hline Model & Equation \\
\hline 1 & $\mathbf{y}_{1}=14.9-145 \mathbf{x}_{13}+475 \mathbf{x}_{14}+123 \mathbf{x}_{15}$ \\
\hline 2 & $\mathbf{y}_{2}=-9.3-218 \mathbf{x}_{13}+418 \mathbf{x}_{14}+145 \mathbf{x}_{15}$ \\
\hline 3 & $\mathbf{y}_{3}=7.6+133 \mathbf{x}_{10}+0.747 \mathbf{x}_{12}+62.2 \mathbf{x}_{15}+0.996 \mathbf{x}_{16}$ \\
\hline 4 & $\mathbf{y}_{4}=-22.5+142 \mathbf{x}_{9}+108 \mathbf{x}_{13}-0.568 \mathbf{x}_{16}$ \\
\hline 5 & $\mathbf{y}_{5}=0.0-162 \mathbf{x}_{10}+162 \mathbf{x}_{14}$ \\
\hline 6 & $\mathbf{y}_{6}=0.0-66.6 \mathbf{x}_{9}+87.4 \mathbf{x}_{10}+66.6 \mathbf{x}_{13}-87.4 \mathbf{x}_{14}$ \\
\hline
\end{tabular}




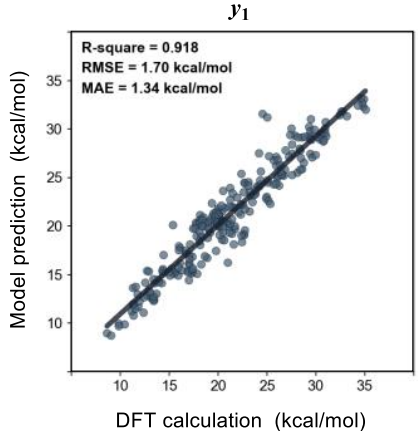

$y_{4}$

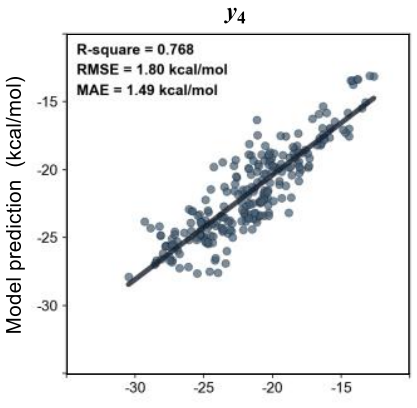

DFT calculation $(\mathrm{kcal} / \mathrm{mol})$
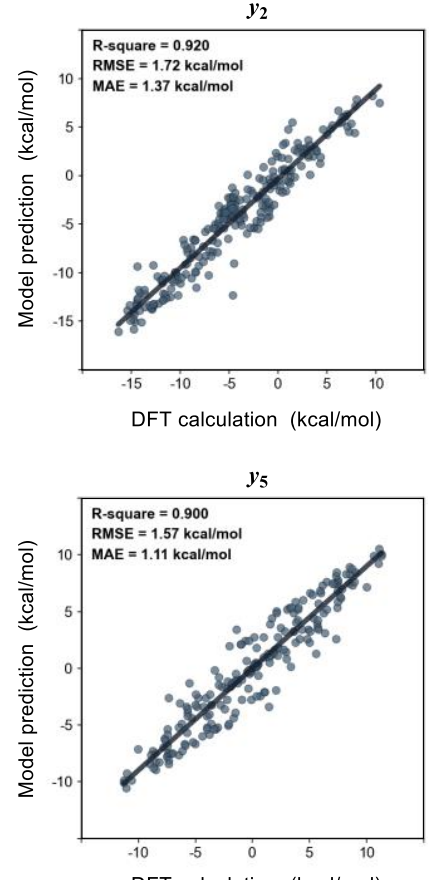

DFT calculation (kcal/mol)
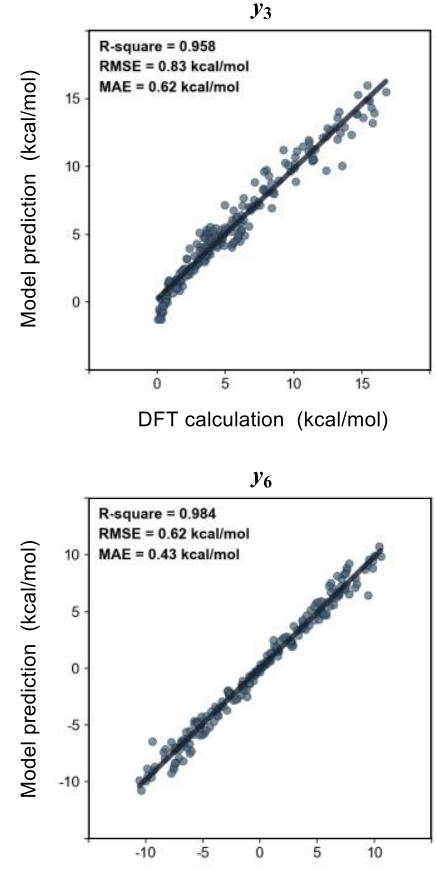

DFT calculation $(\mathrm{kcal} / \mathrm{mol})$

Figure S7. A comparison of the DFT calculated energy and model predicted energy value of the 6 models shown in Table S2.

It is noted that the $\mathrm{R}^{2}$ value for model $\mathrm{y} 4$ is poor. This is possibly due to that only three descriptors were remained after filtering. In other words, the descriptors selected in the agostic species were insufficient to predict y4. Therefore, including more geometrical parameters (such as angle of $\mathrm{C}_{3}-\mathrm{C}_{2}-\mathrm{H}$ and dihedral angle of $\mathrm{Pd}_{-} \mathrm{C}_{3}-\mathrm{C}_{2}-\mathrm{H}$ ) might help improve the performance of this model. Besides, the relationship between the descriptor and response variables might be nonlinear, which may require more complex methods like KRR, SVM or ANN. 


\section{Modeling of the energy profile with propyl chain}

Table S5. The six linear models based on the structure of initial species with methyl group and the energy of the species with a propyl group (one ethylene molecule enchained).

\begin{tabular}{|c|c|}
\hline Model & Equation \\
\hline 1 & $\mathbf{y}_{1}=22.5+1.74 \mathbf{x}_{4}-68.8 \mathbf{x}_{5}+104 \mathbf{x}_{6}-1.49 \mathbf{x}_{8}$ \\
\hline 2 & $\mathbf{y}_{2}=1.7-144 \mathbf{x}_{2}+1.57 \mathbf{x}_{4}-51.4 \mathbf{x}_{5}+127 \mathbf{x}_{6}-0.896 \mathbf{x}_{8}$ \\
\hline 3 & $\mathbf{y}_{3}=5.3-37.9 \mathbf{x}_{1}+59.7 \mathbf{x}_{5}+0.555 \mathbf{x}_{8}$ \\
\hline 4 & $\mathbf{y}_{4}=-34.6+1.92 \mathbf{x}_{4}-261 \mathbf{x}_{7}-3.10 \mathbf{x}_{8}$ \\
\hline 5 & $\mathbf{y}_{5}=0.0-155 \mathbf{x}_{2}+1.96 \mathbf{x}_{4}+155 \mathbf{x}_{6}-1.96 \mathbf{x}_{8}$ \\
\hline 6 & $\mathbf{y}_{6}=0.0-64.5 \mathbf{x}_{1}+112 \mathbf{x}_{2}+64.5 \mathbf{x}_{5}-112 \mathbf{x}_{6}$ \\
\hline
\end{tabular}
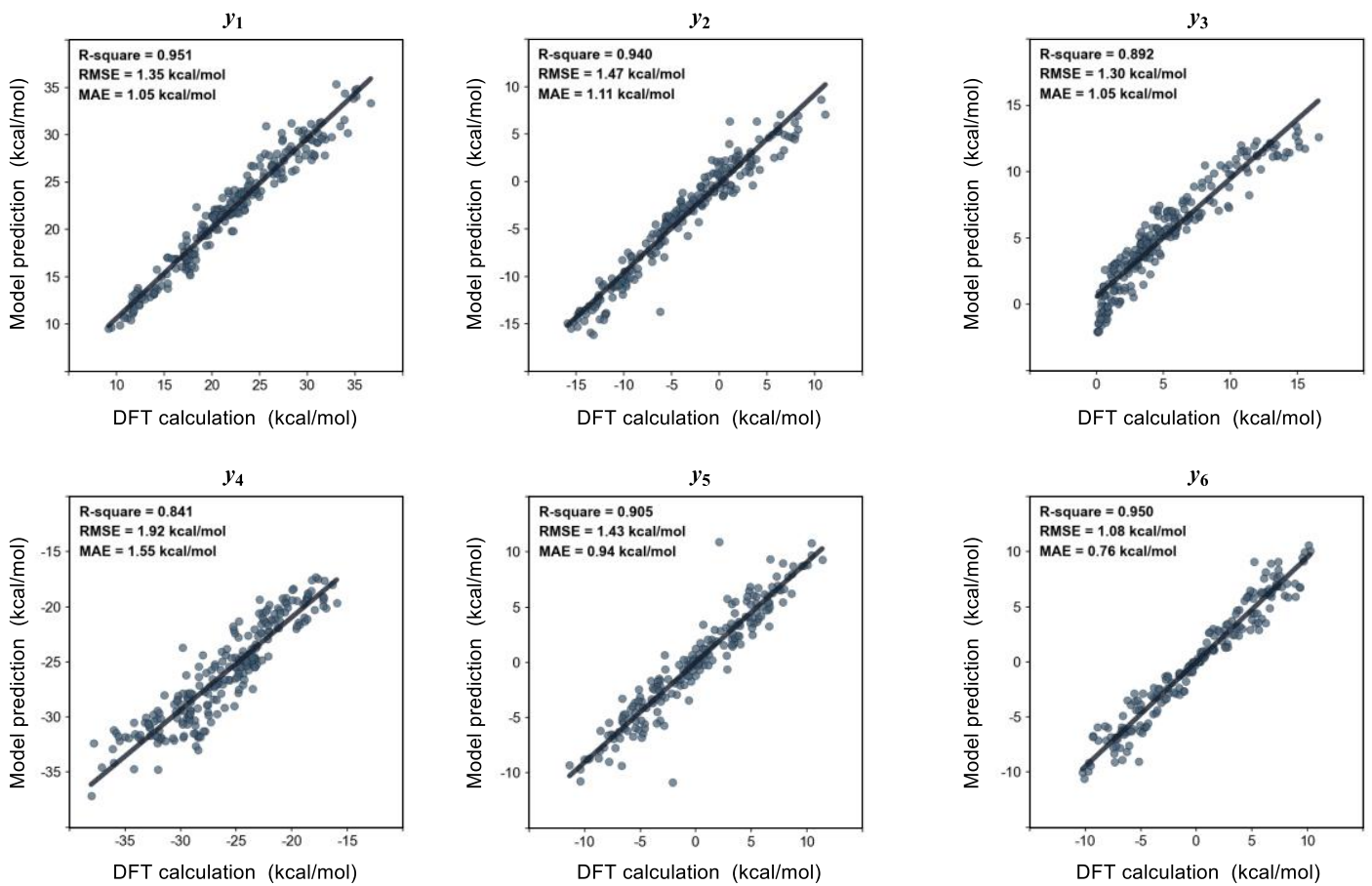

Figure S8. A comparison of the DFT calculated energy and model predicted energy value of 6 models shown in Table S3. 


\section{Electronic effect of ancillary ligands}

Having constructed prediction models with good performance, even for external samples, coefficient analyses of these models were further carried out to obtain chemical knowledge. For this purpose, it is necessary to figure out the electronic effect of ancillary ligands on the geometrical parameters. Such electronic effects include three aspects: (1) electron donation ability of coordinating atoms, (2) ligand backbone connecting the coordinating atoms, and (3) the electronic effect of substitutes. The designed ancillary ligand samples for model construction focus on the first aspect, which will have the greatest influence on the energy data and therefore is the key points in our discussion.

The electronic effects of coordinating atoms and representative structure of coordination complex are shown in Figure S9. In the active species $\mathbf{A}$, alkyl and ethylene interact with $\mathrm{Pd}(\mathrm{II})$ atom through covalent-bond and coordination-bond, respectively. Such interactions are influenced by the ancillary ligands via different manners. The strength of covalent interaction between the alkyl and $\mathrm{Pd}(\mathrm{II})$ is affected by the electron density on the metal center, which is directly influenced by the two coordinating atoms (Figure S9a, left). However, the relatively weak coordinate interaction between ethylene and $\mathrm{Pd}(\mathrm{II})$ is influenced by the coordinating atom being trans to ethylene, namely transsite effect, and the valence-shell electron-pair repulsion of the coordinating atom being cis to ethylene (Figure S9a, right).

The violin plots shown in Figure S9b and S9c depict the effects of coordinating atoms on the geometrical parameters of metal center. The horizontal axis represents various coordinating atoms that follow the order of their electron donation ability, viz., $\mathrm{C}$ (carbene) $\geqslant \mathrm{P}>\mathrm{S} \geqslant \mathrm{N}>\mathrm{Cl} \geqslant \mathrm{O}>\mathrm{F}$. As shown in Figure $\mathrm{S} 9 \mathrm{~b}$, the $\mathrm{Pd}-\mathrm{C}_{1}$ bond length is influenced by the trans atom and the cis atom. When the coordinating atoms are stronger electron donor, the Pd$\mathrm{C}_{1}$ bond tends to elongate. The effects of coordinating atoms on ethylene moiety is shown in Figure 6c. On one hand, the trans coordinating atom affects $\mathrm{Pd}-\mathrm{X}_{\mathrm{dum}}$ and $\mathrm{C}_{2}=\mathrm{C}_{3}$ bond lengths due to the trans effect. When the trans atom have greater electron donation ability, the coordinate interaction will be weakened. As a result, $\mathrm{Pd}-\mathrm{X}_{\mathrm{dum}}$ bond length elongated and the $\mathrm{C}_{2}=\mathrm{C}_{3}$ bond length of ethylene was shortened. On the other hand, the cis atom has an effect on the $\mathrm{C}_{1}-\mathrm{Pd}-\mathrm{X}_{\mathrm{dum}}$ bond angle due to valence-shell electron-pair repulsion. The cis coordinating atom with stronger electron donating ability can increase the repulsion toward ethylene and make it closer to alkyl, which leading to a smaller $\mathrm{C}_{1-}$ $\mathrm{Pd}-\mathrm{X}_{\text {dum }}$ bond angle.

To sum up, in terms of the coordination complex, $\mathbf{x} \mathbf{1} \sim \mathbf{x} 4$ were measured on $\mathbf{A}$ and $\mathbf{x 5} \sim \mathbf{x 8}$ were measured on $\mathbf{A}$ iso. These descriptors will be affected by the electron donation ability of the coordinating atoms. In Figure 4, when the coordinating atom $\mathrm{L}^{1}$ in $\mathbf{A}$ has stronger electron donation ability, the values of $\mathbf{x} \mathbf{1}, \mathbf{x} \mathbf{2}$ and $\mathbf{x} \mathbf{6}$ will be enlarged, but the value of $\mathbf{x} \mathbf{3}$ and $\mathbf{8} \mathbf{8}$ will be reduced. Similarly, when the coordinating atom $\mathrm{L}^{2}$ in $\mathbf{A}$ is a stronger electron donor, the value of $x \mathbf{2}, \mathbf{x} 5$ and $\times \mathbf{6}$ will be enlarged, and the value of $x 7$ and $x 4$ will be reduced. 

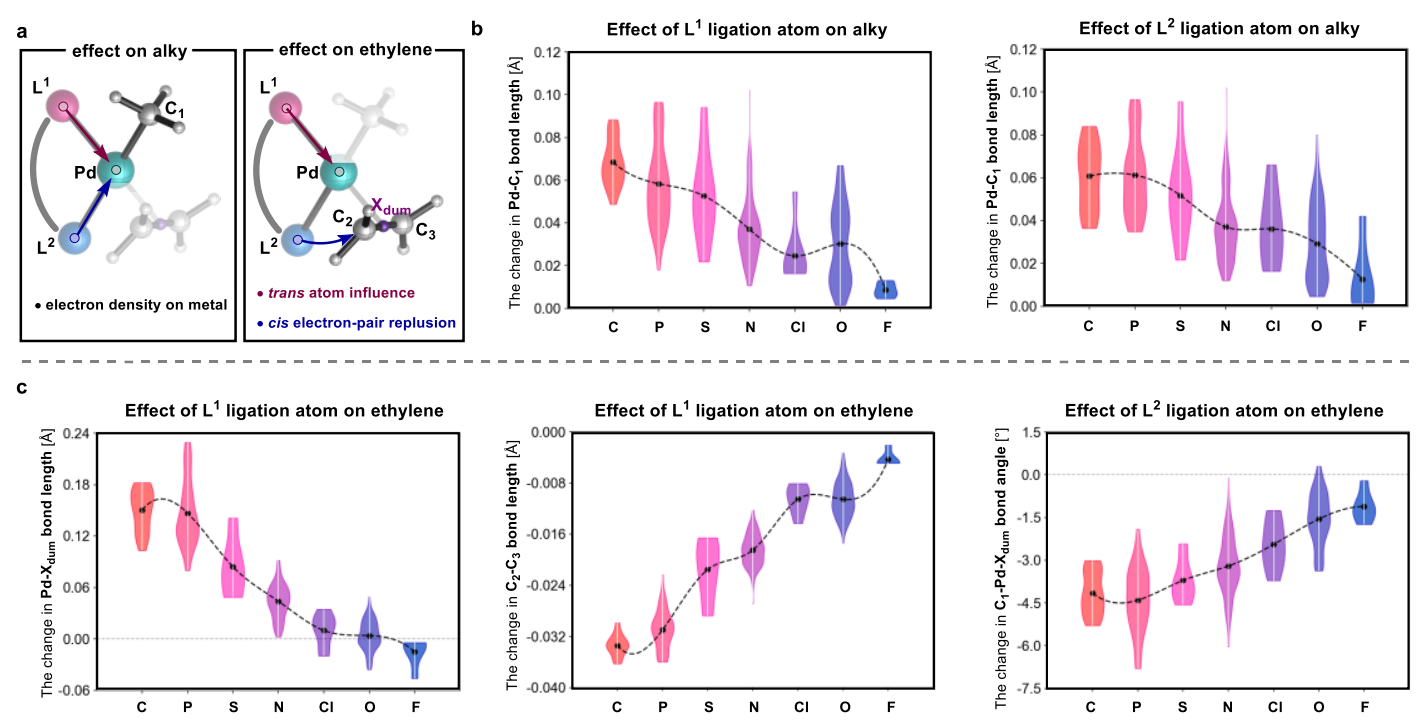

Figure S9. Electronic effect of ancillary ligands on the geometry parameters of the reaction center. a. Electronic effect of ancillary ligands on Pd-alkyl and Pd-ethylene moieties. b. Violin plot of the Pd-alkyl bond length against various trans-and cis- coordinating atoms. c. Violin plot of the change in geometrical parameters that influenced by trans- and cis-coordinating atoms. 


\section{Coefficient analysis of the models}

The regression models are the statistical expression of given data, which can qualitatively and quantitatively afford some common trends via analyzing the coefficient of the models. However, descriptors are different in range and their coefficients have various units. To compare their attributions to the models, we need to multiply coefficient values by standard deviations $(\boldsymbol{\sigma})$ of corresponding descriptors and divide descriptor values by their standard deviations as well. Therefore, in the transformed model, like the form:

$$
\boldsymbol{y}_{i}=\boldsymbol{b}_{i}+\sum_{n} \boldsymbol{c}_{\boldsymbol{i n}}^{\prime} \boldsymbol{x}_{\boldsymbol{n}}^{\prime} \quad(i=1 \sim 6)
$$

where $\mathbf{b}$ was not changed and $\mathbf{c}^{\prime}=\mathbf{c} \boldsymbol{\sigma}, \mathbf{x}^{\prime}=\mathbf{x} / \boldsymbol{\sigma}$. the coefficients $\mathbf{c}^{\prime}$ have the same unit of $\mathrm{kcal} / \mathrm{mol}$ while descriptors $\mathbf{x}^{\prime}$ are non-dimensional. Besides, considering that the descriptors are the difference in geometrical parameters between a given species and the blank sample, the intercepts ( $\mathbf{b i})$ are the energy data of the blank sample predicted by the models. Therefore, in the following discussion, we will use the normalized models and explain how the ancillary ligand changes the energy profile in comparison with the blank sample.

For ethylene insertion, the coefficient analysis of models y 1 and $y \mathbf{5}$ are required. In the case of symmetric ancillary ligands, activity of ethylene insertion from A1 to B1 only correlates with model y1 (Figure S10a). When the two coordination atoms are stronger electron donors $\left(L^{S}-L^{S}\right)$, as aforementioned, the values of $\mathbf{x} \mathbf{4}^{\prime}$ and $\mathbf{x} \mathbf{8}^{\prime}$ are reduced while $\mathbf{x} 5^{\prime}$ and $\mathbf{x} 6^{\prime}$ are enlarged. In the models, the reduced descriptor value together with a positive and negative coefficient will lead to the response variable decrease and increase respectively, while the enlarged descriptor value together with a positive and negative coefficient will lead to the response variable increase and decrease, respectively. In view of this and the sign of coefficients, the reduced $\mathbf{x} 4^{\prime}$ ' (with positive coefficient, $c 4^{\prime}=+2.7$ ) and enlarged $\mathbf{x 5}$ ' (with negative coefficient, $\left.c 5^{\prime}=-4.2\right)$ tend to decrease the energy barrier with the weight of $6.9 \mathrm{kcal} / \mathrm{mol}(2.7+4.2=6.9)$. Similarly, the reduced $\mathbf{x} \mathbf{8}^{\prime}$ ( with negative coefficient, $\mathbf{c 8 ^ { \prime }}=-2.0$ ) and enlarged $\mathbf{x} \mathbf{6}^{\prime}$ ( (with positive coefficient, $\mathbf{c} \mathbf{6}^{\prime}=+2.5$ ) tend to increase the energy barrier with the weight of $4.5 \mathrm{kcal} / \mathrm{mol}(2.0+2.5=4.5)$. According to the intercept and the weights, when the catalyst bearing the ancillary ligand with strong coordinating atoms, the expected value of $y \mathbf{l}$ is $19.1 \mathrm{kcal} / \mathrm{mol}(21.5-6.9+4.5=19.1$, Figure S10c, up). Following the similar analysis for the case of weak coordinating atoms $\left(\mathrm{L}^{\mathrm{W}}-\mathrm{L}^{\mathrm{w}}\right)$, such an expected value is $23.9 \mathrm{kcal} / \mathrm{mol}(21.5+6.9-4.5=23.9)$. This means that the ancillary ligand with strong coordinating atoms is more beneficial to reduce ethylene insertion energy barrier.

In the case of the nonsymmetric ancillary ligand $\left(\mathrm{L}^{\mathrm{S}}-\mathrm{L}^{\mathrm{W}}\right.$ and $\left.\mathrm{L}^{\mathrm{W}}-\mathrm{L}^{\mathrm{S}}\right)$, ethylene coordination and insertion may occur at two sites (from $\mathbf{A} 2$ and $\mathbf{A} 3$, respectively). The complex $\mathbf{A} 2$ either undergoes insertion via $\mathbf{B} 2$ directly or isomerizes to A3 (cis-coordination complex) and complete subsequent insertion process via B3. The ethylene insertion activity in this case is determined by the isomerization energy y 5 (Figure S10b) and the insertion energy barrier y1 (Figure S10a). In these models, as pointed above, the nonsymmetric coordination atoms result in reduced $\mathbf{x} \mathbf{5}^{\prime}$ and $\mathbf{x} \mathbf{8}^{\prime}$, but enlarged $\mathbf{x} \mathbf{4}^{\prime}$ 'when ethylene trans to the stronger electron donor. However, in terms of $\mathbf{x} 2$ ' and $\mathbf{x} \mathbf{6}^{\mathbf{\prime}}$, which could be affected by the two coordinating atoms concurrently. The strong and weak coordinating atoms tend to enlarge and reduce their values, respectively. As a result, the effect of the strong and weak coordinating atoms was counterbalanced and the coefficient ( $c 2$ ' and $\mathbf{c 6}$ ') will not be considered (Figure S10a, b). In model $\mathbf{y} 5$, the positive coefficient of $\mathbf{x} 4^{\prime}$ and negative coefficient of $\mathbf{x} \mathbf{8}^{\prime}$ tend to increase the isomerization energy (from $\mathbf{A 2}$ to $\mathbf{A 3}$ ), with the weight of $7.0 \mathrm{kcal} / \mathrm{mol}$ $\left(3.5+3.5=7.0\right.$, Figure S10c, down). In model $y \mathbf{1}$, the positive coefficient of $x 4^{\prime}$ and negative coefficient of $\mathbf{x} 5^{\prime}$ and $\mathbf{x} \mathbf{8}^{\prime}$ tend to increase the energy barrier between $\mathbf{A} 2$ and $\mathbf{B} 2$ to $30.4 \mathrm{kcal} / \mathrm{mol}(21.5+2.7+4.2+2.0=30.4)$, but decrease the energy barrier between $\mathbf{A} 3$ and $\mathbf{B} 3$ to $12.6 \mathrm{kcal} / \mathrm{mol}(21.5-2.7-4.2-2.0=12.6)$. According to the intercept and 
the weights, in the case of nonsymmetric ligand, the expected value of overall insertion energy barrier along with the favorable pathway $\mathrm{A} 2 \rightarrow \mathrm{A} 3 \rightarrow \mathrm{B} 3$ is $19.6 \mathrm{kcal} / \mathrm{mol}(7.0+12.6)$.

Overall, in comparison with the blank sample, the symmetric ancillary ligands with strong coordinating atom can reduce the insertion energy barrier $(21.5 \mathrm{kcal} / \mathrm{mol}$ for the blank sample vs expected value of $19.1 \mathrm{kcal} / \mathrm{mol})$. In the case of nonsymmetric ancillary ligands, the active species A2, where coordinating ethylene is trans to strong electron donating atom, is more stable. However, the pathway of A2 $\rightarrow$ A3 $\rightarrow$ B3, which features isomerization followed by insertion, is more favorable and has a comparable overall energy barrier to the blank sample $(21.5 \mathrm{kcal} / \mathrm{mol}$ for the blank sample vs expected value of $19.6 \mathrm{kcal} / \mathrm{mol}$ ). The result suggests that the ancillary ligand doesn't change the insertion energy barrier significantly. This also explained the experimental trend that, from the $\mathrm{P}-\mathrm{O}^{3}$ type of nonsymmetric catalysts to the strong electron donated $\mathrm{N}-\mathrm{N}^{1}$ and $\mathrm{P}-\mathrm{P}^{2}$ types of symmetric catalysts, the energy barrier is decreasing but has little difference in values (19.2, 17.2 and $16.6 \mathrm{kcal} / \mathrm{mol}$, respectively).

Similarly, the coefficient analysis of models $\mathrm{y} 3$ and $y 6$ concerning with $\beta$-H elimination has been also carried out. As shown in Figure S11, in the case of symmetric ligand, there is a little difference in energy barrier between the cases of strong and weak coordinating atoms (expected values of 6.1 and $5.1 \mathrm{kcal} / \mathrm{mol} v s 5.6 \mathrm{kcal} / \mathrm{mol}$ for the blank sample, Figure S11c, up). However, in the case of nonsymmetric ancillary ligands, species C3 with alkyl chain trans to the weak coordinating atom is more stable and the direct $\beta$-H elimination $(\mathrm{C} 3 \rightarrow \mathrm{D} 3)$ has an expected value of $12.7 \mathrm{kcal} / \mathrm{mol}$ for the energy barrier of this transformation (Figure S11c, down). Besides, the isomerization-elimination pathway (C3 $\rightarrow \mathrm{C} 2 \rightarrow \mathrm{D} 2)$ has an expected value of more than $8.4 \mathrm{kcal} / \mathrm{mol}$ for the overall energy barrier of this pathway (isomerization from C3 to C2 might have a very high energy barrier). Therefore, the nonsymmetric ancillary ligand can significantly increase the overall energy barrier for $\beta$-H elimination in comparison with the blank sample no matter which pathway it undergone.

Taking the blank sample as a reference, the coefficient analysis revealed the different effect of symmetric and nonsymmetric ancillary ligands. Such a coefficient analysis reflected average effect of the ligands on the energy as the parameters were normalized. The catalysts bearing symmetric ligands with strong coordinating atoms like $\alpha$-diimine ${ }^{1}$ is known to get a highly branched polymer, while the catalysts bearing nonsymmetric ligands like phosphine-sulfonate ${ }^{4}$ is known to get a linear polymer. Such a difference is caused by the competition of ethylene insertion and $\beta$-H elimination. The symmetric catalyst with strong coordinating atoms is possible to slightly reduce the energy barrier of ethylene insertion and $\beta$-H elimination. However, the nonsymmetric catalysts have close insertion energy barrier with the symmetric catalysts, but the energy barrier for $\beta$-hydrogen elimination is expected to be higher. As a result, ethylene tends to insert continuously and obtain linear polymer with high molecular weight. 

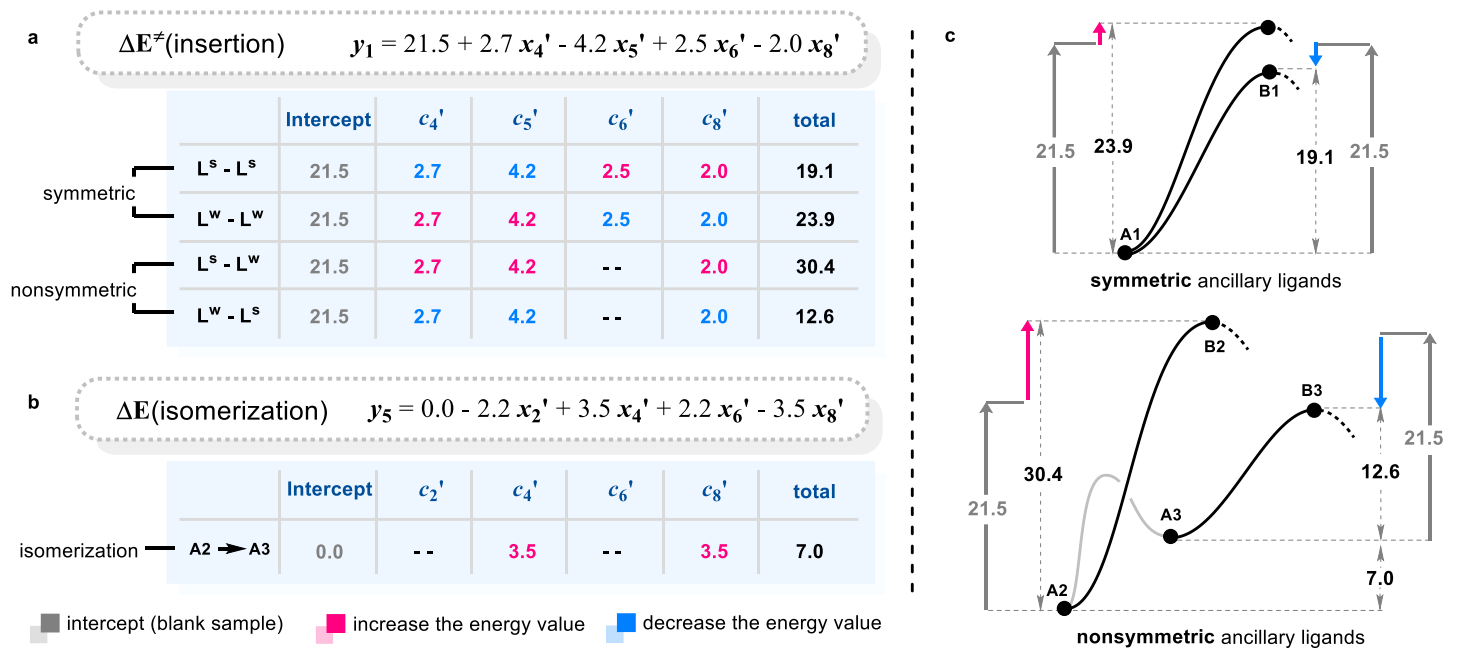

Figure S10. Coefficient analysis on ethylene insertion. a. Effect of ancillary ligands on ethylene insertion. The values in red and blue indicate that the corresponding term leads the energy value increase and decrease, respectively. $\mathbf{b}$. Effect of ancillary ligands on the isomerization from $\mathbf{A} 2$ to $\mathbf{A} 3$. c. Overall mechanism of ethylene insertion with electronic symmetric (up) and nonsymmetric (down) ancillary ligands. The energy data are statistic values on the basis of the intercept and coefficients of the models. $\mathrm{L}^{\mathrm{S}}$ and $\mathrm{L}^{\mathrm{W}}$ labels in the ancillary ligand represent the strong and weak electron donors, respectively.

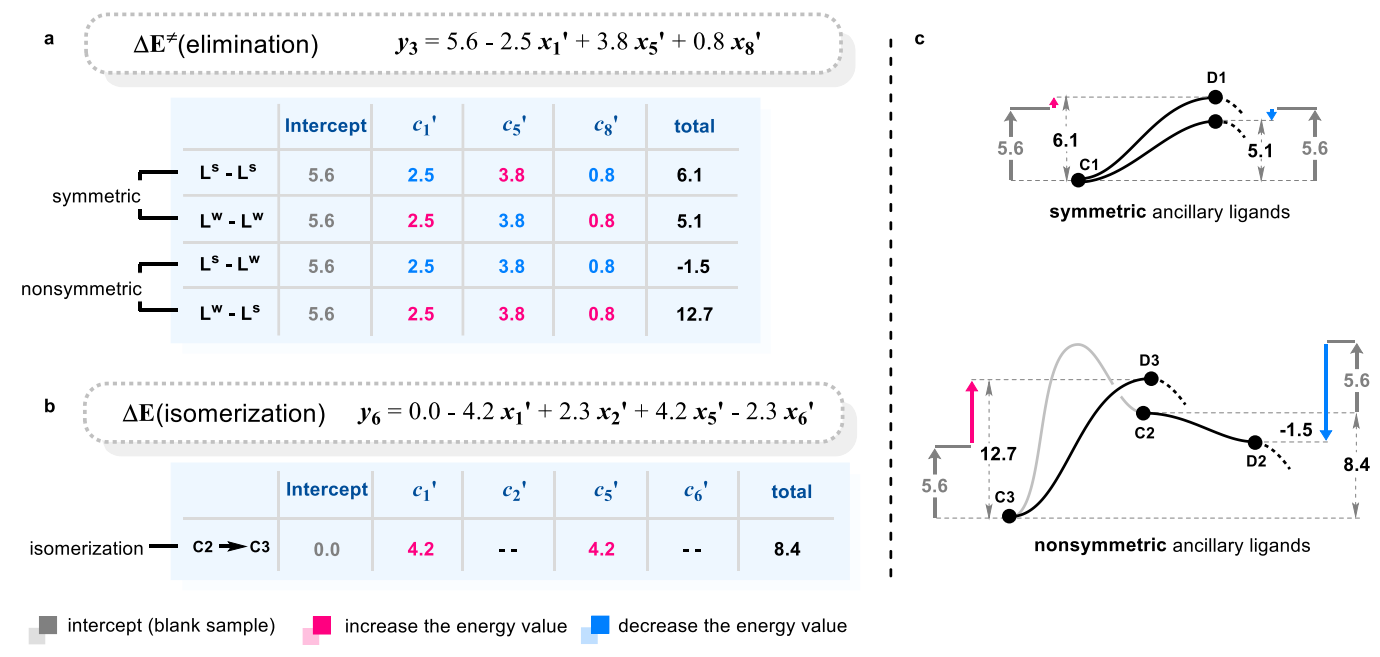

Figure S11. Coefficient analysis on $\beta$-H elimination. a. Effect of ancillary ligands on $\beta$-H elimination. The value in red and blue indicate that the term will lead to the energy data increase and decrease, respectively. $\mathbf{b}$. Effect of ancillary ligands on the isomerization from $\mathbf{C} 2$ to $\mathbf{C} 3$. c. Overall mechanism of $\beta$-H elimination with electronic symmetric (up) and nonsymmetric (down) ancillary ligands. The energy data are statistic values on the basis of the intercept and coefficients of the models. $L^{S}$ and $L^{\mathrm{W}}$ labels in the ancillary ligand represent the strong and weak electron donors, respectively. 


\section{Discussion of the symmetric of the ancillary ligands}

According to aforementioned model analyses, nonsymmetric ligand tends to give linear and high molecular weight polymer. For example, replacing the phosphine part in phosphine-sulfonate catalysts by a stronger donor NHC (Nheterocyclic carbene) could greatly improve the molecular weight of resulting polymer. ${ }^{5}$ It is noteworthy that, however, by replacing the sulfonate part in phosphine-sulfonate catalysts with weaker donor trifluoroborate, only dimer rather than polymer was obtained. ${ }^{6,7}$ According to our calculation, the weak electron donor F atom in trifluoroborate could be favorably replaced by stronger $\mathrm{N}$ atom in lutidine to coordinate to the metal center, which weakened the electron nonsymmetry effect of the catalyst (from P-F type to P-N type, Figure S12). As a result, the free energy barrier of $\beta$ hydrogen elimination was significantly reduced from $22.1 \mathrm{kcal} / \mathrm{mol}$ to $12.2 \mathrm{kcal} / \mathrm{mol}$ while ethylene insertion barrier was slightly reduced from $21.7 \mathrm{kcal} / \mathrm{mol}$ to $21.4 \mathrm{kcal} / \mathrm{mol}$, leading to the preference of $\beta$-hydrogen elimination over ethylene insertion and thus to give the dimer product. Therefore, to enlarge the electron nonsymmetry effect of a catalyst, enhancing the electron donation ability of strong coordinating atom is superior to weakening the electron donation ability of weak coordinating atom.

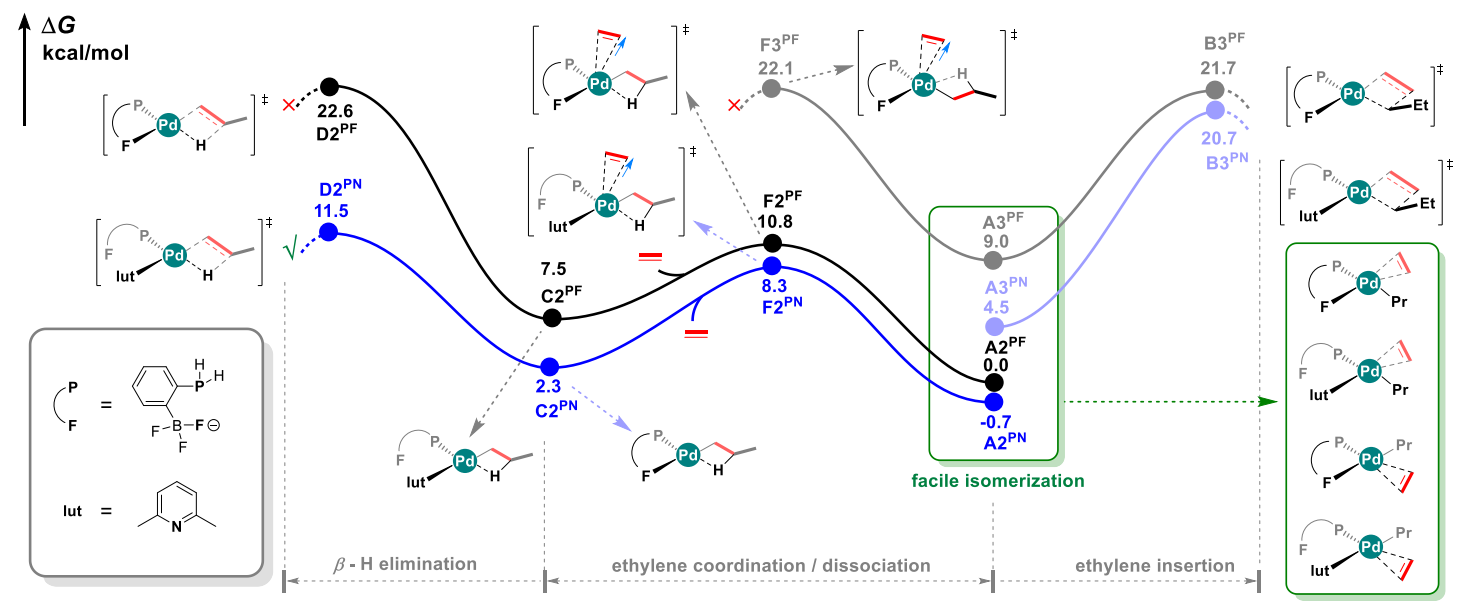

Figure S12. Mechanism of phosphine-trifluoroborate type catalyst for ethylene polymerization. 


\section{Discussion of the ligand backbone}

In the current dataset of ancillary ligands, some samples having strong and weak donors between which only one carbon unit (samples 075, 133 and 136, see Figure S13) exhibited the largest energy barrier difference between $\beta$ hydrogen elimination and ethylene insertion. This is probably due to that the short spacer between the two coordinating atoms endows the ligand backbone with rigidity and is against the coordination of the weak coordinating atom. In this situation, the electron nonsymmetry effect could be enhanced. However, such an insufficient chelation could result in de-coordination of the weak coordinating atom, and the solvent or additive may coordinate to the metal center instead, like the case of P-F type ancillary ligand (Figure S12).

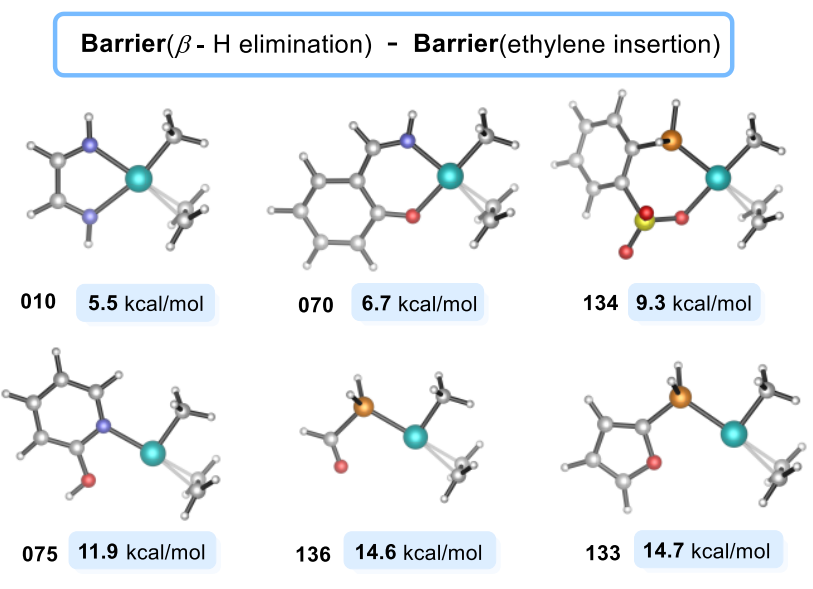

Figure S13. Energy barrier difference between $\beta$-hydrogen elimination and ethylene insertion of typical catalysts (010, 070, $134)$ and catalysts with four-membered ring that have more advantage in ethylene insertion $(075,133,136)$. 


\section{Reference}

(1) Johnson, L. K.; Killian, C. M.; Brookhart, M. New Pd(II)- and Ni(II)-Based Catalysts for Polymerization of Ethylene and $\alpha$ Olefins. J. Am. Chem. Soc. 1995, 117, 6414-6415.

(2) Shultz, C. S.; Ledford, J.; DeSimone, J. M.; Brookhart, M. Kinetic Studies of Migratory Insertion Reactions at the (1,3Bis(diphenylphosphino)propane)Pd(II) Center and Their Relationship to the Alternating Copolymerization of Ethylene and Carbon Monoxide. J. Am. Chem. Soc. 2000, 122, 6351-6356.

(3) Malinoski, J. M.; Brookhart, M. Polymerization and Oligomerization of Ethylene by Cationic Nickel(II) and Palladium(II) Complexes Containing Bidentate Phenacyldiarylphosphine Ligands. Organometallics 2003, 22, 5324-5335.

(4) Drent, E.; van Dijk, R.; van Ginkel, R.; van Oort, B.; Pugh, R. I. Palladium Catalysed Copolymerisation of Ethene with Alkylacrylates: Polar Comonomer Built into the Linear Polymer Chain. Chem. Commun. 2002, 744-745.

(5) Nakano, R.; Nozaki, K. Copolymerization of Propylene and Polar Monomers Using Pd/IzQO Catalysts. J. Am. Chem. Soc. 2015, $137,10934-10937$.

(6) Gott, A. L.; Piers, W. E.; Dutton, J. L.; McDonald, R.; Parvez, M. Dimerization of Ethylene by Palladium Complexes Containing Bidentate Trifluoroborate-Functionalized Phosphine Ligands. Organometallics 2011, 30, 4236-4249.

(7) Kim, Y.; Jordan, R. F. Synthesis, Structures, and Ethylene Dimerization Reactivity of Palladium Alkyl Complexes That Contain a Chelating Phosphine-Trifluoroborate Ligand Organometallics 2011, 30, 4250-4256. 